\title{
LESÕES TRAUMÁTICAS DO MEDIASTINO: ASPECTOS NA TOMOGRAFIA COMPUTADORIZADA*
}

\author{
Alessandro Severo Alves de Melo ${ }^{1}$, Luiza Beatriz Melo Moreira², Edson Marchiori ${ }^{3}$
}

Resumo As lesões traumáticas mediastinais constituem achados pouco freqüentes no trauma torácico, mas são de extrema importância, por representarem mau prognóstico, dada a sua gravidade e lesões associadas. 0 trauma mediastinal é cada vez mais diagnosticado pela tomografia computadorizada, em especial pelo rápido tempo de aquisição decorrente da técnica helicoidal, que permite a avaliação de pacientes em estado grave, possibilitando a adoção de conduta terapêutica eficiente. Os autores estudaram 11 pacientes com trauma torácico submetidos a tomografia computadorizada, os quais apresentaram lesões mediastinais. A hemorragia mediastinal representou a lesão mais comum nesta casuística, manifestando-se sob a forma de infiltração da gordura mediastinal, com a presença de material denso permeando os espaços mediastinais, ou sob a forma de hematoma. 0 hemopericárdio representou a segunda lesão mais comum, caracterizado por material denso ou líquido no interior do pericárdio. As lesões aórticas foram observadas em três casos, caracterizadas por irregularidade parietal no contorno aórtico ou por pseudo-aneurisma. Neste trabalho o trauma torácico fechado foi observado em seis casos, enquanto o trauma aberto ocorreu em cinco pacientes. As causas de trauma fechado foram atropelamento, colisão automobilística e queda de altura. A forma penetrante de traumatismo torácico decorreu de dois modos de agressão: lesão por arma de fogo e lesão por arma branca

Unitermos: Hemorragia mediastinal; Lesão da aorta; Tomografia computadorizada.

Abstract Traumatic mediastinal lesions: computed tomography findings.

Traumatic mediastinal lesions are unusual findings in patients with thoracic trauma but may represent poor prognosis and be associated with severe lesions. Mediastinal trauma is increasingly being diagnosed by computed tomography due to the fast acquisition times of helical techniques, allowing the evaluation of critically ill patients and the adoption of efficient therapeutic measures. The authors studied 11 patients with mediastinal lesions who were submitted to computed tomography due to thoracic trauma. The most frequent finding was mediastinal bleeding characterized by infiltration of mediastinal fat, dense material within the mediastinal spaces or hematoma. Hemopericardium was the second most common lesion in this series, appearing as dense or liquid material in the pericardium. Aortic lesions such as wall irregularities or pseudoaneurisms were less commonly seen (three patients). In this study blunt thoracic traumas were observed in six cases whereas penetrating trauma occurred in five cases. The causes of blunt trauma were pedestrians struck by car, motor vehicle accidents and falls. Penetrating trauma was due to lesions produced by bullets or knifes.

Key words: Mediastinal bleeding; Aortic lesion; Computed tomography.

\section{INTRODUÇÃO}

O trauma é uma das principais causas de morte e invalidez no mundo atual, em especial na população mais jovem, e a le-

* Trabalho realizado no Serviço de Radiologia do Hospital Municipal Souza Aguiar (HMSA), Rio de Janeiro, RJ.

1. Médico Radiologista do HMSA e do Hospital Barra D'Or, Doutorando em Radiologia pela Universidade Federal do Rio de Janeiro (UFRJ).

2. Médica Radiologista do HSMA, Mestre em Radiologia pela UFR.

3. Professor Titular de Radiologia da Universidade Federal Fluminense (UFF), Coordenador Adjunto do Curso de Pós-Graduação em Radiologia da UFRJ.

Endereço para correspondência: Dr. Alessandro Severo Alves de Melo. Rua Cinco de Julho, 273, ap. 1302, Icaraí. Niterói, RJ, 24220-110. E-mail: Lubiamoreira@bol.com.br

Recebido para publicação em 20/2/2003. Aceito, após revisão, em 14/4/2003. são torácica representa agravante importante na evolução dos pacientes com trauma multissistêmico, chegando a determinar $20 \%$ das mortes de origem traumática ${ }^{(\mathbf{1})}$.

O uso da tomografia computadorizada (TC) tem representado um significativo avanço na abordagem moderna das vítimas de trauma. A TC avalia muito bem as lesões traumáticas do crânio e face, coluna, tórax, abdome e pelve, permitindo um melhor estudo das lesões dos pacientes traumatizados e, conseqüentemente, desempenhando papel essencial nos centros hospitalares especializados ${ }^{(\mathbf{1})}$.

A TC, especialmente a TC helicoidal, apresenta maior sensibilidade e especificidade que as radiografias convencionais
(RX) de tórax na detecção e avaliação da extensão das lesões traumáticas do parênquima pulmonar, do espaço pleural, da aorta e do diafragma, possibilitando melhor avaliação das lesões, e também permitindo o diagnóstico correto das lesões extratorácicas $^{(2-7)}$.

Neste trabalho são discutidas as principais alterações encontradas na TC de 11 pacientes com lesão mediastinal traumática, e realizada revisão da literatura sobre o assunto.

\section{MATERIAIS E MÉTODOS}

Foram reunidos, em um estudo prospectivo, 11 casos de lesão traumática de 
mediastino, diagnosticados no período de janeiro/2001 a janeiro/2003, provenientes de três instituições do Rio de Janeiro, RJ: Hospital Municipal Souza Aguiar, Hospital Barra D’Or e Hospital Copa D’Or.

A faixa etária dos pacientes variou de 20 a 74 anos, com média de idade de 44,2 anos. O sexo masculino predominou, com cerca de $81,8 \%(n=9)$, sendo apenas dois pacientes $(18,2 \%)$ do sexo feminino.

Os pacientes foram submetidos a TC do tórax em aparelhos de aquisição helicoidal com $5 \mathrm{~mm}$ de colimação, da fúrcula esternal até o andar superior do abdome, sendo os exames realizados até 12 horas após o trauma torácico.

$\mathrm{Na}$ análise dos casos deste trabalho os aspectos estudados foram o tipo do trauma torácico, sendo dividido em trauma fechado e aberto, as causas das lesões traumáticas, determinadas por acidente automobilístico, atropelamento, arma de fogo, queda de altura e lesão por arma branca, e o padrão tomográfico das lesões.

\section{RESULTADOS}

As alterações mediastinais foram determinadas por trauma torácico fechado em seis casos $(54,5 \%)$ e por trauma torácico aberto ou penetrante em cinco pacientes $(45,5 \%)$. Em relação ao trauma fechado houve predomínio do atropelamento, fator causal em três pacientes $(27,2 \%$ do total geral de traumas torácicos mediastinais), seguido pela colisão de automóveis em dois casos $(18,1 \%)$ e queda de altura em um caso $(9 \%)$. A forma aberta ou penetran- te de traumatismo torácico decorreu de dois modos de agressão: a lesão por arma de fogo, presente em quatro casos $(36,3 \%$ do total geral de traumas mediastinais), e a lesão por arma branca, observada em um caso $(9 \%)$.

A hemorragia mediastinal foi a lesão mais comum nesta casuística, estando presente em sete pacientes $(63,6 \%)$, manifestando-se, na TC, sob a forma de infiltração da gordura mediastinal, com a presença de material denso permeando os espaços mediastinais, ou sob a forma de hematoma (Figura 1).

O hemopericárdio representou a segunda lesão mais comum neste trabalho, observado em quatro pacientes, correspondendo a 36,3\% dos casos, sendo caracterizado por material denso ou líquido no interior do pericárdio (Figura 2).

As lesões aórticas foram observadas em três casos $(27,2 \%)$, caracterizadas por irregularidade parietal no contorno aórtico (Figuras 3 e 4) em dois casos ou por pseudo-aneurisma traumático em um caso (Figura 5). Todos estes casos estiveram associados a hemorragia mediastinal.

As lesões mediastinais foram observadas sempre em associação com lesões de outros compartimentos torácicos.

Os outros sítios torácicos acometidos foram: o parênquima pulmonar em todos os casos (sob a forma de contusões em dez pacientes, atelectasias em cinco e áreas de laceração em um) e o espaço pleural (hemotórax em nove casos $(81,8 \%)$ e pneumotórax em cinco casos $(45,4 \%))$. Enfisema de partes moles e pneumomediastino estiveram presentes em um caso, correspondendo a $9 \%$ do total de casos. Lesões diafragmáticas não foram observadas.

\section{DISCUSSÃO}

O trauma representa uma das principais causas de invalidez e morte na atualidade, afetando uma população jovem, predominantemente masculina ${ }^{(6-10)}$. Em nossa casuística de trauma torácico com lesão mediastinal associada também foi observado um maior número de pacientes do sexo masculino, correspondendo a $81,8 \%$ dos $\operatorname{casos}(\mathrm{n}=9)$.

Um estudo institucional multicêntrico sobre trauma, realizado nos $\mathrm{EUA}^{(\mathbf{5})}$, demonstrou predomínio do trauma fechado sobre o trauma penetrante. No nosso estudo, seis pacientes foram vítimas de trauma torácico fechado, representando 54,5\%, enquanto cinco pacientes sofreram trauma penetrante $(45,5 \%)$.

$\mathrm{Na}$ literatura o acidente automobilístico é a causa mais comum de trauma torácico, variando entre $70 \%$ e $85 \%$ do total de $\operatorname{casos}^{(\mathbf{6 , 9 )}}$. Na nossa casuística as lesões por arma de fogo predominaram com quatro casos, representando cerca de $36,4 \%$, seguidas pelo atropelamento, presente em três casos $(27,2 \%)$.

Atualmente, com o advento da TC helicoidal, tem sido sugerido seu uso no diagnóstico precoce do trauma torácico ${ }^{(1-3)}$. $\mathrm{O}$ valor da TC no estudo das lesões neurológicas e abdominais já está bem estabelecido, enquanto a utilização deste método para o trauma de tórax, por sua intro-

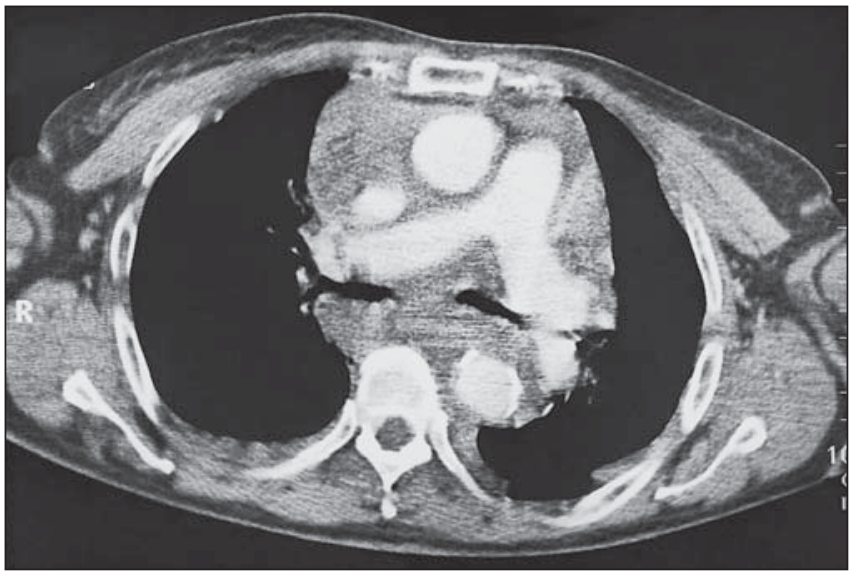

Figura 1. Hemorragia mediastinal, caracterizada por grande quantidade de materialdenso (sangue) infiltrando o mediastino. Pequeno hemotórax bilateral.

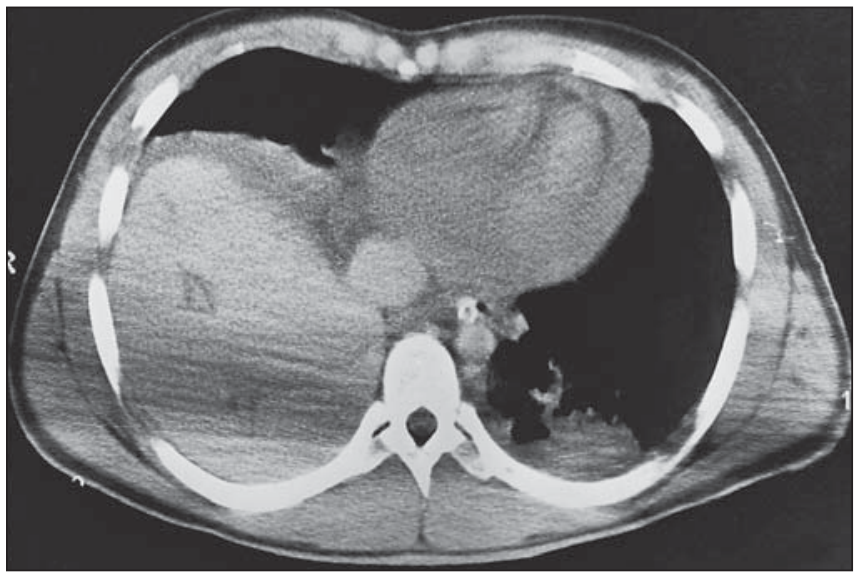

Figura 2. Líquido pericárdico espontaneamente denso, caracterizando hemopericárdio. Área de consolidação no lobo inferior esquerdo. Hemoperitônio. 


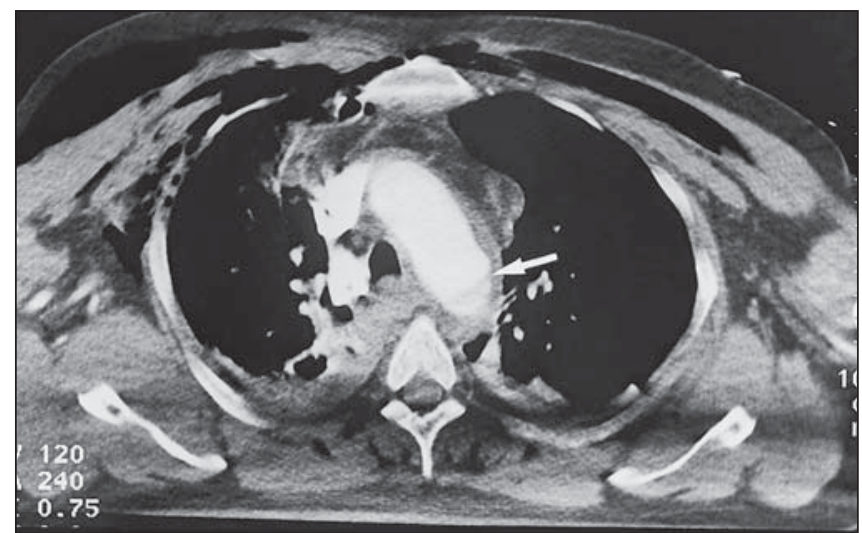

A

Figura 3. Em A, irregularidade parietal na croça da aorta (seta), associada a hemorragia mediastinal e hemotórax bilateral. Enfisema de partes moles. Em B a lesão aórtica é visualizada no plano sagital (seta).

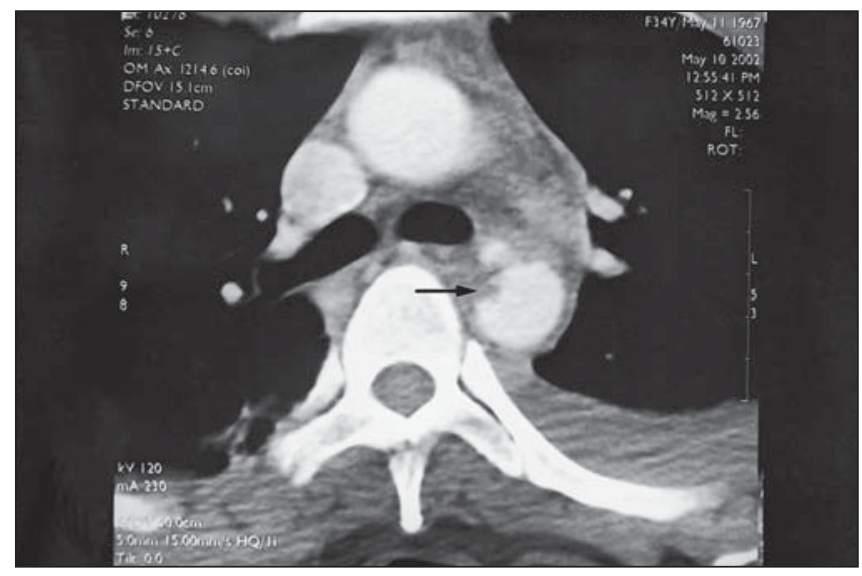

Figura 4. Pequeno entalhe na aorta torácica descendente (seta), com hemorragia mediastinal e hemotórax bilateral, causado por acidente automobilístico.

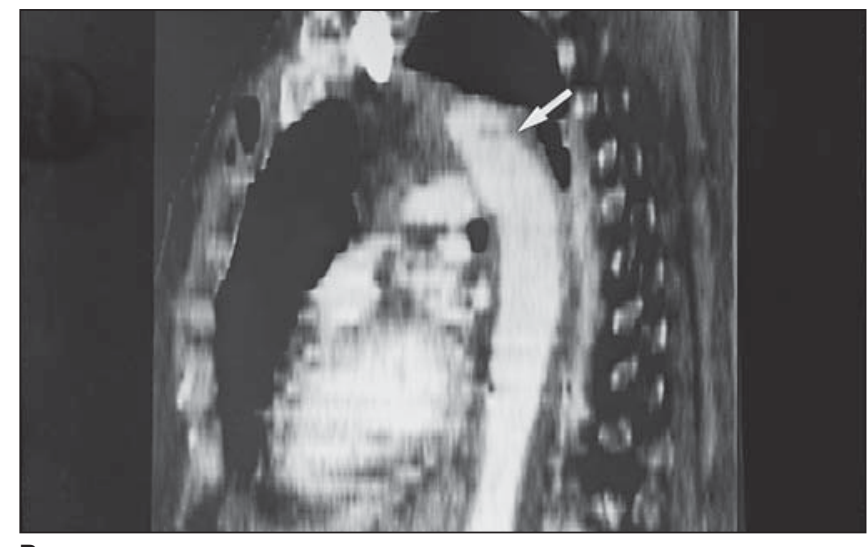

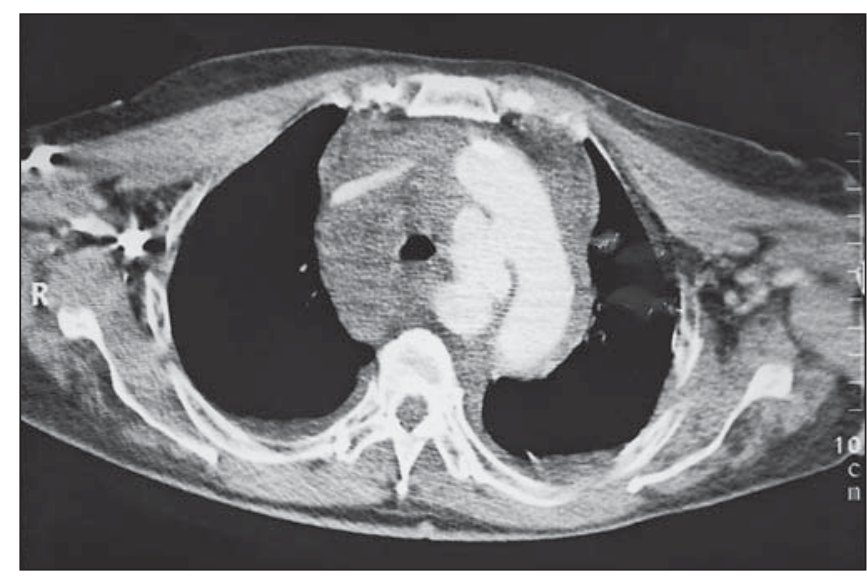

Figura 5. Volumoso pseudo-aneurisma roto do arco aórtico, associado a grande hemorragia mediastinal e a pequeno hemotórax bilateral. dução recente, permanece um campo a ser desenvolvido $^{(\mathbf{1}-\mathbf{3})}$.

A TC do tórax, nos casos de trauma torácico, define mais precisamente a extensão e a gravidade das lesões, que geralmente são subestimadas pelos $\mathrm{RX}^{(\mathbf{7 , 8 )}}$. Esta observação também é verdadeira no que se refere às lesões mediastinais, conforme demonstrado por Karaaslan et al. ${ }^{(7)}$ e Demetriades et al. ${ }^{(2)}$.

Karaaslan et al. $^{(7)}$ afirmam que a TC foi importante para a mudança de conduta, através de dados adicionais fornecidos em $12,7 \%$ dos casos, tendo demonstrado lesão aórtica em um paciente, confirmada pela arteriografia e cirurgia, com associação de hematoma mediastinal.

Em estudo prospectivo feito por Demetriades et al. ${ }^{(2)}$, com 112 pacientes vítimas de trauma, nove pacientes sofreram ruptura da aorta, sendo que quatro apresentavam mediastino normal nos RX, e o diagnóstico da lesão aórtica foi feito pela TC. A sensibilidade e a especificidade do RX para este diagnóstico foram de $55,5 \%$ e $64,1 \%$, respectivamente, enquanto a TC teve sensibilidade de $100 \%$ e especificidade de $95,1 \%$. Em 42 pacientes o mediastino foi considerado alargado, do ponto de vista radiológico. Destes, somente cinco pacientes $(11,9 \%)$ apresentavam lesão de aorta. Um terço dos pacientes com lesão aórtica apresentava ruptura de aorta com RX normal.

As lesões traumáticas mediastinais decorrem de trauma grave, em geral associado a alta mortalidade, cursando com lesões de outros compartimentos do tórax e de outros sítios corporais, como o crânio e o abdome $^{(2,3)}$. Tal observação foi feita no nosso estudo, em que houve associação com lesões pulmonares ou pleurais em todos os casos.
A hemorragia mediastinal se manifesta por má definição da gordura mediastinal, com acúmulo de material denso no seu interior, ou por lesão expansiva densa (hematoma), podendo decorrer de sangramentos de pequenos vasos arteriais ou venosos, ou até de extensas lesões aórticas ${ }^{(2,3,11)}$. No nosso estudo, hemorragia mediastinal esteve presente em sete casos; em três destes foi detectada evidência tomográfica de lesão da aorta e nos demais não se identificou nenhuma lesão vascular à TC.

O hemopericárdio manifesta-se pela presença de sangue no saco pericárdico, que na TC é caracterizado pelo achado de material denso ou líquido circundando o coração, podendo ser observado nos casos de trauma torácico fechado ou aberto ${ }^{(11)}$. O hemopericárdio pode acompanhar lesões traumáticas cardíacas, de coronárias, da aorta e de outros sítios torácicos ${ }^{(11)}$. No 
nosso estudo, em todos os casos esteve acompanhado de outras lesões torácicas pulmonares ou pleurais. Clinicamente, pode estar associado a tamponamento cardíaco $^{(11)}$.

A ruptura ou laceração da aorta torácica é um dos mais dramáticos e temidos resultados do trauma de tórax ${ }^{(\mathbf{1 2})}$. As lesões de aorta resultam em $80 \%$ dos óbitos por trauma de tórax, por sua associação com contusão miocárdica, ruptura de valva aórtica, laceração de artéria coronária e hemopericárdio $^{(\mathbf{1 2})}$. Cerca de $70 \%$ dos pacientes com trauma de aorta morrem no local do acidente, e de $80 \%$ a $90 \%$ antes de serem transportados para o hospital ${ }^{(\mathbf{1 2})}$. A importância crucial do seu diagnóstico precoce está na necessidade de adotar conduta terapêutica adequada, seja com cirurgia, seja com tratamento clínico específico ${ }^{(3)}$. No nosso trabalho houve óbito de dois dos três pacientes com trauma aórtico.

A TC helicoidal tem sido usada cada vez mais no intuito de firmar este diagnóstico nos pacientes vítimas de trauma torácico $^{(6)}$. As lesões da aorta se manifestam na TC por irregularidades parietais ou pseudo-aneurismas e ocorrem, em cerca de $90 \%$ dos casos, no istmo, distal à origem da artéria subclávia esquerda, representando o ponto de máximo estresse mecâni$\mathrm{co}^{(6)}$. No nosso estudo observamos a presença de lesão aórtica nesta topografia em dois casos. Apenas 5\% das lesões aórticas acometem a porção descendente da aorta $^{(6)}$, sítio da lesão em um de nossos casos.

Em estudo de Dyer et al. ${ }^{(3)}$, com 1.009 pacientes com suspeita clínica de lesão aórtica, 802 foram examinados pela TC helicoidal, que mostrou sensibilidade e valor preditivo negativo de $100 \%$, tendo estabelecido o diagnóstico tomográfico em 152 pacientes, o que correspondeu a $19 \%$ dos casos estudados. Esses autores referem que os mecanismos de injúria principais nos casos de suspeita de lesão aórtica foram acidente automobilístico (779 casos), quedas (83 casos), atropelamento (69 casos) e acidentes de motocicleta $(50 \text { casos })^{(3)}$. No nosso estudo, acidente automobilístico foi o fator causal em dois dos três casos.

No estudo de Demetriades et $a l^{(2)}$, todos os pacientes foram submetidos a TC de crânio, tendo sido encontradas lesões em 87 pacientes $(77,7 \%)$, e 77 pacientes realizaram TC de abdome (68,8\% do total), sendo que em 25 casos alguma anormalidade foi encontrada. Ainda nesse estudo, 45 pacientes $(40,2 \%)$ foram submetidos à TC de coluna, sendo detectadas 11 fraturas $^{(2)}$. Dois dos nossos pacientes apresentaram fraturas de costela e clavícula, e um deles, pneumoperitônio.

Dyer et al. ${ }^{(3)}$ encontraram hematoma mediastinal e periaórtico como sinais importantes para o diagnóstico de lesão de aorta, apresentando valor preditivo negativo de quase $100 \%$. Este sinal foi observado no nosso trabalho em todos os casos.

A TC deve ser considerada como exame prévio à angiografia, por este ser um exame invasivo, dispendioso e de difícil utilização como rotina ${ }^{(2)}$, enquanto a TC é acessível, de rápida execução, de mais baixo custo, além de poucos centros terem a possibilidade de realizar aortografia tão rápido como a $\mathrm{TC}^{(3)}$. A $\mathrm{TC}$ pode ainda detectar a presença de outras lesões significativas, como pneumotórax, hemopericárdio e fraturas vertebrais ${ }^{(\mathbf{9})}$. No estudo de Demetriades et al. ${ }^{(2)}$, três pacientes foram levados à cirurgia com base no diagnóstico tomográfico, sem aortografia prévia, sendo sugerido, por esses autores, a substituição deste método pela TC.

Demetriades et al. $^{(\mathbf{2})}$ e Greene ${ }^{(\mathbf{1 3})}$ sugerem que pacientes com lesões de desaceleração ou lesões associadas a aumento súbito de pressão intratorácica devem ser submetidos a TC, em função destes mecanismos de trauma poderem acarretar lesão aórtica $^{(2,3)}$.

Dyer et al. ${ }^{(3)}$ afirmam que os pacientes com suspeita clínica de lesão aórtica devem inicialmente ser submetidos a TC helicoidal, estando indicada aortografia nos casos em que houver hematoma periaórtico e sinais equívocos de lesão aórtica na TC. Os pacientes que apresentam lesão aórtica evidente na TC devem ser submetidos a aortografia ou a cirurgia imediata, considerando que, segundo esses autores, a TC é capaz de reproduzir uma aortografia adequada para o planejamento cirúrgico.

Demetriades et al. ${ }^{(2)}$ demonstraram nove casos de lesão de aorta confirmada pela TC e dez casos com lesão de aorta diagnosticada pela toracotomia ou necropsia. Estes números, segundo esses autores, provavelmente subestimam os dados reais, posto que consideram que a maioria dos pacientes com lesão traumática da aorta sequer chega ao hospital, falecendo no local do trauma. Os autores consideram, ainda, que os pacientes que chegam ao hospital e são submetidos a TC apresentam rupturas contidas ou pequenas ${ }^{(2)}$.

$\mathrm{O}$ nosso estudo permite concluir que a TC do tórax, nas vítimas de trauma, representa um avanço significativo, por sua capacidade diagnóstica, devendo tornar-se método rotineiro da avaliação do paciente politraumatizado nos centros hospitalares que possuem aparelhos helicoidais.

\section{REFERÊNCIAS}

1. Cárdenas GP, Marchiori E, Campos ACL, Macedo JRC, Henrique K, Magalhães MR. Hematoma mediastinal por lesão venosa traumática. Radiol Bras 1996;29:41-4.

2. Demetriades D, Gomez H, Velmahos GC, et al. Routine helical computed tomographic evaluation of the mediastinum in high-risk blunt trauma patients. Arch Surg 1998;133:1084-8.

3. Dyer DS, Moore EE, Mestek ME, et al. Can chest CT be used to exclude aortic injury? Radiology 1999;213:195-202.

4. Feliciano DV. Patterns of injury. In: Feliciano DV, Moore EE, Mattox KL, eds. Trauma. 3rd ed. Stanford: Appleton \& Lange, 85-103.

5. Feliciano DV, Rozycki GS. Advances in the diagnosis and treatment of thoracic trauma. Surg Clin North Am 1999;79:1417-29.

6. Groskin SA. Selected topics in chest trauma. Radiology 1992;183:605-17.

7. Karaaslan T, Meuli R, Androux R, Duvoisin B, Hessler C, Schnyder P. Traumatic chest lesions in patients with severe head trauma: a comparative study with computed tomography and conventional chest roentgenograms. J Trauma 1995;39:1081-6.

8. LoCícero J 3rd, Mattox KL. Epidemiology of chest trauma. Surg Clin North Am 1989;69:15-9.

9. Marts B, Durham R, Shapiro M, et al. Computed tomography in the diagnosis of blunt thoracic trauma. Am J Surg 1994;168:688-92.

10. Tambelini AT, Osanai CH. Epidemiologia do trauma. In: Freire E. Trauma - a doença do século. $1^{\text {a }}$ ed. São Paulo, SP: Atheneu, 2001:47-76.

11. Toombs BD, Sandler CM, Lester RG. Computed tomography of chest trauma. Radiology 1981;140: 733-8.

12. McGonigal MD, Schwab CW, Kauder DR, Miller WT, Grumbach K. Supplemental emergent chest computed tomography in the management of blunt torso trauma. J Trauma 1990;30:1431-4.

13. Greene R. Blunt thoracic trauma. Radiological Society of North America. RSNA Publications 1992:297-309 